\title{
All-cause mortality of metabolically healthy or unhealthy obese: risk stratification using myocardial perfusion imaging
}

Andrea De Lorenzo ${ }^{1,2}$, Mariana Carazza², Ronaldo Lima ${ }^{1,3,4}$

\author{
${ }^{1}$ Universidade Federal do Rio de Janeiro, Rio de Janeiro, Brazil \\ 2Instituto Nacional de Cardiologia, Rio de Janeiro, Brazil \\ ${ }^{3}$ Clinica de Diagnostico por Imagem, Rio de Janeiro, Brazil \\ ${ }^{4}$ Clinica Fonte Imagem, Rio de Janeiro, Brazil
}

Submitted: 22 April 2018

Accepted: 20 June 2018

Arch Med Sci Atheroscler Dis 2018; 3: e90-e95

DOI: https://doi.org/10.5114/amsad.2018.76865

Copyright $\odot 2018$ Termedia \& Banach

\author{
Corresponding author: \\ Andrea De Lorenzo \\ Universidade Federal \\ do Rio de Janeiro \\ Av Brigadeiro Trompowsky \\ 22210085 Rio de Janeiro, \\ Brazil \\ Phone: +21995316994 \\ Fax: +21 995316994 \\ E-mail: andlorenzo@hotmail. \\ com
}

\begin{abstract}
Introduction: There is still controversy about the favorable prognosis of "metabolically healthy" ( $\mathrm{MH})$ obese. This study evaluated mortality and the use of myocardial perfusion scintigraphy (MPS) for risk stratification of $\mathrm{MH}$ or metabolically unhealthy (MU) obese or nonobese patients.

Material and methods: Patients without dyslipidemia, hypertension, or diabetes were considered $\mathrm{MH}$, and those with $\geq 1$ of these risk factors were considered MU. The MPS was categorized as normal, abnormal or ischemic. Patients were followed for $4.0 \pm 1.0$ years for all-cause death.

Results: Of 2450 patients, 613 were obese. The MH obese patients less often had ischemia than MU obese, but there was no significant difference in the prevalence of ischemia compared to all nonobese. The annualized death rate of $\mathrm{MH}$ obese was $1.3 \%$ and of nonobese $1.0 \%(p=0.4)$. An abnormal MPS and the MU status were independently associated with death, with hazard ratios of 1.85 and 1.72 , respectively. A normal MPS identified patients with low risk among all subgroups; annualized rates of death were $1.0 \%, 1.1 \%$ and $1.0 \%$ for all nonobese, $\mathrm{MH}$ obese and $\mathrm{MU}$ obese, respectively $(p=0.63)$. Conclusions: The annualized death rate of $\mathrm{MH}$ obese patients was not significantly different from that of nonobese individuals. Myocardial perfusion scintigraphy was able to stratify prognosis in the overall patient population. These data may be helpful to identify high-risk individuals, thereby improving patient management.
\end{abstract}

Key words: obesity, metabolically healthy obesity, myocardial perfusion SPECT, coronary artery disease, prognosis.

\section{Introduction}

Obesity is a major health concern, associated with a wide range of diseases, from cardiovascular to cancer [1]. However, not all obese individuals have the same metabolic or risk factor profile. Individuals without insulin resistance, lipid disorders, or hypertension have been called "metabolically healthy" ( $\mathrm{MH})$ obese [2]. These are considered to have a risk of cardiovascular disease and mortality that is intermediate between $\mathrm{MH}$ nonobese individuals and metabolically unhealthy (MU) obese adults $[3,4]$. Nonetheless, this concept is controversial, as the "benign" nature of $\mathrm{MH}$ obesity has not been definitely established [5]. 
The noninvasive assessment of cardiovascular prognosis has an important role in the care of patients with increased baseline risk, such as the obese, as a more refined evaluation may allow more individualized management. Myocardial perfusion scintigraphy (MPS) has been employed for over 2 decades [6], has well-established prognostic value [7], and has undergone technological advances that have enabled its use in populations previously considered to present limitations for imaging, such as the obese [8]. The study of obesity phenotypes with MPS may therefore cast additional light on the discussion regarding $\mathrm{MH}$ obesity.

\section{Material and methods}

\section{Study population}

Subjects $(n=3476)$ without known coronary artery disease (defined as a history of myocardial infarction, myocardial revascularization by coronary angioplasty or bypass surgery, or the presence of known obstructive epicardial coronary disease detected by coronary computed tomography or coronary angiography and treated medically) were selected among 4737 patients who underwent MPS for clinically indicated reasons at a single center in Rio de Janeiro, Brazil, between 2011 and 2015. Those with nonischemic cardiomyopathies, significant valvular heart disease or who had early revascularization after MPS (< 60 days) were excluded $(n=$ 540). From the remainder, $16.5 \%$ were lost to follow-up, leaving a population of 2450 patients, followed for $4 \pm 1$ years.

Demographic and clinical variables were ascertained at the time of the MPS study by patient interview and review of medical records. Obesity was defined as a body mass index (BMI) $\geq 30 \mathrm{~kg} /$ $\mathrm{m}^{2}$. Patients were assigned according to the World Health Organization into the following categories: normal weight (BMI: $\left.18.5-24.9 \mathrm{~kg} / \mathrm{m}^{2}\right)$, overweight (BMI: $25-29.9 \mathrm{~kg} / \mathrm{m}^{2}$ ), obesity class I (BMI: $30-34.9 \mathrm{~kg} / \mathrm{m}^{2}$ ), class II (BMI: $\left.35-39.9 \mathrm{~kg} / \mathrm{m}^{2}\right)$, or class III (BMI $\left.\geq 40 \mathrm{~kg} / \mathrm{m}^{2}\right)$ [9].

Individuals were considered metabolically healthy $(\mathrm{MH})$ if they had none of the following: dyslipidemia, considered as a history of hypercholesterolemia, hypertriglyceridemia or low HDL, alone or in combination (defined by self-report plus the referring physician's report or the prescription of specific medications for the control of blood lipids); systemic hypertension, defined by history or use of antihypertension medication; or diabetes, considered as a history of diabetes with the use of hypoglycemic medication. Metabolically unhealthy (MU) individuals were considered as those with at least 1 of these 3 risk factors (diabetes, hypertension or dyslipidemia).
The research complies with the Declaration of Helsinki and was approved by the institutional review board, and written informed consent was obtained from all patients.

\section{Myocardial perfusion scintigraphy}

Patients underwent treadmill exercise or pharmacological stress using standard dipyridamole or dobutamine infusion protocols [10]. Exercise testing was performed using a symptom-limited Bruce protocol. A 1-day, rest/stress protocol was used, with injection of 222-370 MBq of Tc-99m sestamibi at rest and 666-1110 MBq at stress. The injected radiotracer activity was scaled according to the patient's weight. For rest studies, < 80 kg: $222 \mathrm{MBq}$; 80-90 kg: $259 \mathrm{MBq}$; 91-100 kg: $296 \mathrm{MBq}$; 101-110 kg: 333 MBq; > 110 kg: 370 MBq. Poststress prone acquisitions were performed in all patients. For stress studies, injected activity was 3 times the rest dose.

MPS was performed with a CZT-SPECT system (Discovery 530, GE Healthcare, Milwaukee, USA) using previously described protocols [8, 11]. Images were reconstructed on a dedicated Xeleris workstation (GE Healthcare). Assessment of perfusion abnormalities was performed using combined supine and prone images, by two experienced nuclear cardiologists blinded to patient characteristics, who employed a standard 5-point scoring system for semiquantitative visual interpretation of myocardial perfusion images, generating summed stress and rest scores (SSS and SRS), while their difference was recorded as the summed difference score (SDS). Discrepancies in perfusion scores between the readers were resolved by consensus. Patients with SSS $\geq 3$ were considered to have abnormal MPS, while patients with SDS > 1 were considered to have ischemic MPS. Post-stress left ventricular ejection fraction (LVEF) was calculated from the post-stress gated images using commercially available software (QGS, Cedars-Sinai Medical Center, Los Angeles, California, USA).

\section{Follow-up and outcomes}

The primary outcome of the study was allcause death. Vital status was ascertained by integrating data from telephone interviews performed every 6 months (up to May 2016) and medical records.

\section{Statistical analysis}

Continuous variables were expressed as mean \pm standard deviation or median and interquartile range and were compared by Student's $t$ test. Categorical variables were expressed as number and percentage and compared by the $\chi^{2}$ 
or Fisher's exact test. Kaplan-Meier curves were constructed to analyze survival free of all-cause death and were compared with a log-rank test. A Cox proportional hazards analysis was performed to identify, among demographic, clinical and MPS variables, those independently associated with all-cause death, and to obtain the risk-adjusted hazards ratio of baseline (clinical and scintigraphic) factors associated with all-cause death. Analyses were performed with SPSS software, version 20.0. A $p$-value $<0.05$ was considered statistically significant.

\section{Results}

Of 2450 patients, 613 (25.0\%) were obese, of whom 413 (67.4\%) were class I, 120 (19.6\%) class II, and 80 (13.0\%) class III. In addition, 829 (33.8\%) of the patients were overweight, and therefore $58.9 \%$ were above normal weight. The proportion of $\mathrm{MH}$ subjects was lower in obese patients than in nonobese $(13.3 \%$ vs. $25.0 \%, p<0.001)$. Demographic, clinical and MPS variables of $\mathrm{MH}$ or MU obese patients are shown in Table I. The $\mathrm{MH}$ phenotype was predominantly found in class I obese patients. $\mathrm{MH}$ patients were younger, more frequently in obesity class I or II and more frequently able to exercise than MU patients, while they less often had ischemic MPS studies. When

Table I. Comparison between metabolically healthy and metabolically unhealthy obese patients

\begin{tabular}{|c|c|c|}
\hline Parameter & $\begin{array}{c}\text { MH obese } \\
(n=82)\end{array}$ & $\begin{array}{c}\text { MU obese } \\
(n=531)\end{array}$ \\
\hline Age [years] & $55.3 \pm 13.6$ & $60.8 \pm 11.2^{*}$ \\
\hline Male & $43(52.4)$ & $262(49.3)$ \\
\hline BMI $\left[\mathrm{kg} / \mathrm{m}^{2}\right]$ & $33.9 \pm 4.7$ & $34.9 \pm 5.1$ \\
\hline Obesity class I & $61(74.4)$ & $350(65.9)^{*}$ \\
\hline Obesity class II & $14(17.1)$ & $110(20.7)^{*}$ \\
\hline Obesity class III & $7(8.5)$ & $71(13.4)^{*}$ \\
\hline Diabetes & & $193(36.3)$ \\
\hline Hypertension & & 467 (87.9) \\
\hline Dyslipidemia & & $310(58.4)$ \\
\hline Smoking & $22(26.8)$ & $201(37.8)$ \\
\hline Typical angina & $1(1.2)$ & $11(2.1)$ \\
\hline Exercise stress & $67(59.8)$ & $247(46.5)^{*}$ \\
\hline Abnormal MPS & $10(12.1)$ & $73(13.7)$ \\
\hline Ischemic MPS & 9 (10.9) & $79(14.9)^{\star}$ \\
\hline LVEF (\%) & $61.6 \pm 9.2$ & $59.9 \pm 10.0$ \\
\hline
\end{tabular}

Numbers are $n(\%)$ or mean \pm standard deviation. ${ }^{*} p<0.05$. BMI - body mass index, LVEF - left ventricular ejection fraction, MPS myocardial perfusion scintigraphy.
$\mathrm{MH}$ obese patients were compared to nonobese individuals (Table II), the former were younger and less often able to exercise. However, there were no significant differences regarding the prevalence of abnormal MPS or of ischemic MPS.

\section{Outcomes}

The annualized death rate of $\mathrm{MH}$ obese patients was $1.3 \%$ and of nonobese $1.0 \%(p=0.4)$. A normal MPS study was able to identify patients with low risk among all subgroups; annualized rates of death were $1.0 \%, 1.1 \%$ and $1.0 \%$ for all nonobese, $\mathrm{MH}$ obese and $\mathrm{MU}$ obese, respectively $(p=0.63)$. Overall, an abnormal MPS study and the MU status (but not BMI) were independently associated with death, with hazard ratios of 1.85 (95\% Cl: $1.23-2.79)(p=0.003)$ and $1.72(95 \% \mathrm{Cl}$ : $1.02-3.1)(p=0.04)$, respectively (Figure 1$)$.

\section{Discussion}

Obesity is a global problem of increasing magnitude which is associated with and a contributor to several chronic pathological states, with cardiovascular disease being one of the most frequent and serious [12]. A subgroup of obese individuals, referred to as "metabolically healthy", has been considered of lower cardiovascular risk, closer to normal weight individuals; however, its true phenotype, definitions and risk of incident cardiovascular disease are still a matter of debate [13-22]. Therefore, further data on the prognosis of this

Table II. Comparison between metabolically healthy obese and all nonobese patients

\begin{tabular}{|lcc|}
\hline Parameter & $\begin{array}{c}\text { MH obese } \\
(n=82)\end{array}$ & $\begin{array}{c}\text { Nonobese } \\
(n=1837)\end{array}$ \\
\hline Age [years] & $55.3 \pm 13.6$ & $63.9 \pm 12.2^{\star}$ \\
\hline Male & $43(52.4)$ & $866(47.1)$ \\
\hline BMI [kg/m²] & $33.9 \pm 4.7$ & $25.4 \pm 2.6^{*}$ \\
\hline Diabetes & & $320(17.4)$ \\
\hline Hypertension & $1048(57.0)$ \\
\hline Dyslipidemia & $1(1.2)$ & $914(49.8)$ \\
\hline Smoking & $22(26.8)$ & $645(35.1)$ \\
\hline Typical angina & $67(59.8)$ & $1159(63.1)^{\star}$ \\
\hline Exercise stress & $10(12.1)$ & $207(11.3)$ \\
\hline Abnormal MPS & $9(10.9)$ & $154(8.4)$ \\
\hline Ischemic MPS & $61.6 \pm 9.2$ & $61.0 \pm 9.5$ \\
\hline LVEF (\%) & & $23)$ \\
\hline
\end{tabular}

Numbers are $n(\%)$ or mean \pm standard deviation. ${ }^{*} p<0.05 . B M I$ - body mass index, LVEF - left ventricular ejection fraction, MPS myocardial perfusion scintigraphy. 
subgroup are valuable to understand its pathophysiology as well as to identify high-risk individuals who merit more intensive management. This study has searched for further evidence in a cohort of patients who underwent MPS, which offers the additional opportunity to evaluate the use of this noninvasive imaging method for risk stratification of this patient population.

In this study, the $\mathrm{MH}$ phenotype was present in $13.3 \%$ of the obese patients. Studies indicate that $10-30 \%$ of obese adults can be categorized as $\mathrm{MH}[2,20,21]$, but the definitions of $\mathrm{MH}$ obesity are very heterogeneous [14-16, 22], limiting the comparisons among studies. Obese individuals, especially if $M U$, were less frequently able to exercise than those without obesity, as previously described $[23,24]$ underscoring that regular engagement in physical activity should be reinforced, especially because increased physical activity tends to offset the increased cardiovascular mortality seen in obese populations [24, 25].

Within the obese population, the MU status was associated with a higher prevalence of ischemic MPS, as expected due to the clustering of cardiovascular risk factors [26]. However, when $\mathrm{MH}$ obese individuals were compared to nonobese, the prevalence of ischemic MPS was not significantly different, as previously demonstrated [27]. It should be noted that while in our prior study we observed that class III obese patients, even when $\mathrm{MH}$, had an increased prevalence of myocardial ischemia compared to nonobese [27], in the current study we did not assess class III obese patients individually due to their small number.

Despite the similar prevalence of ischemic MPS studies between $\mathrm{MH}$ obese or nonobese individuals, other factors, such as inability to exercise or obesity itself, with its cluster of associated abnormalities, might influence outcomes [14-16, 28-30]. Thus, the prognostic capacity of MPS widely employed in several patient populations over the last decades [31] - is well suited for risk stratifying this patient subgroup. In this study, the overall annualized rates of all-cause death of $\mathrm{MH}$ obese or all nonobese patients were not significantly different. When mortality predictors were evaluated, the MU status and an abnormal MPS test were found to be independently associated with all-cause death. A normal MPS scan was associated with similar mortality rates $(\sim 1 \%)$ year) across all patient subgroups ( $\mathrm{MH}$ obese, $\mathrm{MU}$ obese or nonobese), as previously demonstrated in other populations [31]. Of note, BMI was not independently associated with death. Johnson et al. [32], in a study using myocardial perfusion imaging, showed an inverse impact of BMI on mortality, after controlling for confounding variables. This "obesity paradox" is another contro-

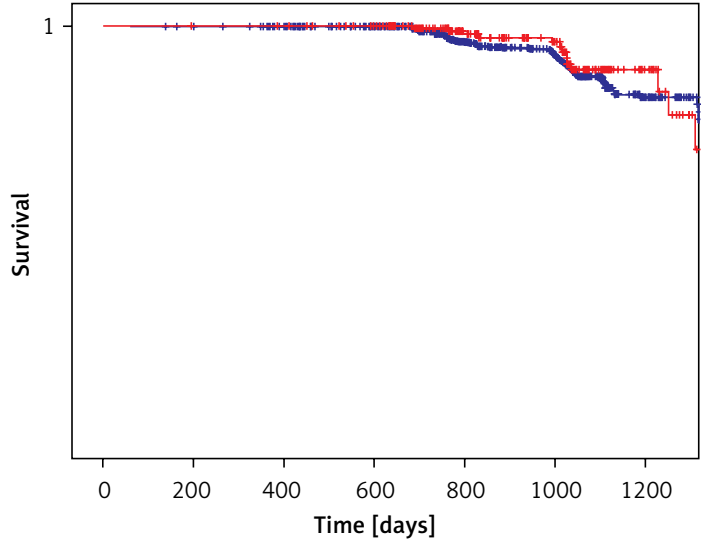

Figure 1. Kaplan-Meier curves showing survival free of all-cause death in nonobese (blue line) or metabolically healthy obese (red line) individuals. Value of $p=0.63$ for the comparison between curves

versial issue, suggesting that "moderate" obesity may in fact be protective, and only higher values of BMI (as well as low BMI) negatively impact survival $[33,34]$. Our findings suggest that metabolic risk factors are more important predictors of all-cause death than BMI, which is in line with recent data from a large study of several cohorts [35]. Thus, the current results are consistent with the $\mathrm{MH}$ obesity concept. Unfortunately, due to the small number of class III obese patients, we were not able to investigate whether higher BMI, despite the MH status, would negatively impact survival, following the "obesity paradox" [33].

Nonetheless, several points should be raised. When the follow-up is longer, increased risk associated with MH obesity may be found [36]. The $\mathrm{MH}$ obesity is possibly a transitional state, from "apparently healthy" to "evidently sick" [37, 38]. Additionally, obesity is associated with several medical complications [39], and weight loss should be targeted to prevent these nonmetabolic diseases. Also, obesity itself is a chronic, progressive disorder, which worsens unless treated. Finally, a healthy metabolic profile is desirable, irrespective of BMI.

This study has limitations. This is a single-center study whose population is composed of outpatients without known coronary artery disease, which may drive cardiovascular risk towards lower levels. If patients with coronary artery disease were included, mortality rates would be likely higher, the predictors of death would be different, and $\mathrm{MH}$ obesity might have a different impact on mortality as well. However, we chose to study patients without known coronary artery disease because the disease itself is closely linked to prognosis - therefore, the influence of the $\mathrm{MH}$ phenotype on prognosis might be overcome by the presence of coronary artery disease. 
Additionally, biochemical data were not available for all patients, and therefore our definition of metabolic health included the components of the metabolic syndrome which could be most easily recorded during the patient interview before the MPS study, based on history plus medical reports or drug prescriptions. However, that approach is similar to a previous large study [40]. It is worth noting that knowledge on different patterns of obesity and its associations with metabolic disorders and prognosis keeps evolving, including new biomarkers such as microRNAs, which have been able to distinguish metabolically healthy from unhealthy obese [41].

On the other hand, and also to be kept in mind, our definition of $\mathrm{MH}$ considered the absence of dyslipidemia, hypertension or diabetes, which is a "stricter" criterion (compared to other studies which allowed the $\mathrm{MH}$ definition to consist of 1 risk factor) and might be a better definition of this condition, as it eliminates the "contamination" of data by any of the abovementioned risk factors. This might also have approximated the mortality rate of $\mathrm{MH}$ obese to that of nonobese, differently from other recent studies [5].

In conclusion, all-cause mortality rates were not significantly different between metabolically healthy obese and all nonobese individuals, supporting the concept of the "metabolically healthy obese". Myocardial perfusion scintigraphy was able to stratify prognosis in the overall patient population, being an independent predictor of death when abnormal and defining low mortality when normal. The metabolically unhealthy status was also associated with death, underscoring the need for continuous efforts to improve the metabolic profile of obese individuals. These data may be helpful for the phenotyping of obese persons, which is important both to understand the pathophysiology of metabolic disturbances in obesity and to identify high-risk individuals or subgroups, thereby improving patient management.

\section{Conflict of interest}

The authors declare no conflict of interest.

\section{References}

1. Kopelman PG. Obesity as a medical problem. Nature 2000; 404: 635-43.

2. Bluher M. The distinction of metabolically 'healthy' from 'unhealthy' obese individuals. Curr Opin Lipidol 2010; 21: 38-43.

3. Bell JA, Kivimaki M, Hamer M. Metabolically healthy obesity and risk of incident type 2 diabetes: a metaanalysis of prospective cohort studies. Obes Rev 2014; 15: 504-15.

4. Kramer CK, Zinman B, Retnakaran R. Are metabolically healthy overweight and obesity benign conditions?
A systematic review and meta-analysis. Ann Intern Med 2013; 159: 758-69.

5. Lassale C, Tzoulaki I, Moons KGM, et al. Separate and combined associations of obesity and metabolic health with coronary heart disease: a pan-European case-cohort analysis. Eur Heart J 2018; 39: 397-406.

6. Maddahi J, Kiat H, Van Train KF, et al. Myocardial perfusion imaging with technetium-99m sestamibi SPECT in the evaluation of coronary artery disease. Am J Cardiol 1990; 66: 55E-62E.

7. Berman DS, Hachamovitch R, Kiat $\mathrm{H}$, et al. Incremental value of prognostic testing in patients with known or suspected ischemic heart disease: a basis for optimal utilization of exercise technetium-99m sestamibi myocardial perfusion single-photon emission computed tomography. J Am Coll Cardiol 1995; 26: 639-47.

8. De Lorenzo A, Peclat T, Amaral AC, Lima RSL. Prognostic evaluation in obese patients using a dedicated multipinhole cadmium-zinc telluride SPECT camera. Int J Cardiovasc Imaging 2016; 32: 355-61.

9. World Health Organization. BMI classification. http:// apps.who.int/bmi

10. Henzlova MJ, Cerqueira MD, Hansen CL, Mahmarian JJ, Yao SS. ASNC imaging guidelines for nuclear cardiology procedures: stress protocols and tracers. J Nucl Cardiol 2009; 16: 331-61.

11. Lima R, De Lorenzo A, Camargo G, et al. Prognostic value of myocardium perfusion imaging with a new reconstruction algorithm. J Nucl Cardiol 2014; 21: 149-57.

12. www.who.int/topics/obesity. Accessed on Sept $27^{\text {th }}$, 2017.

13. Calori G, Lattuada G, Piemonti L, et al. Prevalence, metabolic features, and prognosis of metabolically healthy obese Italian individuals: the Cremona Study. Diabetes Care 2011; 34: 210-5.

14. Ogorodnikova AD, Kim M, McGinn AP, Muntner P, Khan U, Wildman RP. Incident cardiovascular disease events in metabolically benign obese individuals. Obesity (Silver Spring) 2012; 20: 651-9.

15. Hamer M, Stamatakis E. Metabolically healthy obesity and risk of all-cause and cardiovascular disease mortality. J Clin Endocrinol Metabol 2012; 97: 2482-8.

16. Stefan N, Haring HU, Hu FB, Schulze MB. Metabolically healthy obesity: epidemiology, mechanisms, and clinical implications. Lancet Diabetes Endocrinol 2013; 1: 152-62.

17. Bluher M. Are metabolically healthy obese individuals really healthy? Eur J Endocrinol 2014; 171: R209-19.

18. Karelis AD. To be obese - does it matter if you are metabolically healthy? Nat Rev Endocrinol 2011; 7: 699-700.

19. Wildman RP, Muntner P, Reynolds K, et al. The obese without cardiometabolic risk factor clustering and the normal weight with cardiometabolic risk factor clustering: Prevalence and correlates of 2 phenotypes among the US population (NHANES 1999-2004). Arch Intern Med 2008; 168: 1617-24.

20. Bluher M. The distinction of metabolically 'healthy' from 'unhealthy' obese individuals. Curr Opin Lipidol 2010; 21: 38-43.

21. Garcia-Moll X. Obesity and prognosis: time to forget about metabolically healthy obesity. Eur Heart J 2018; 39: 407-9.

22. Caleyachetty R, Thomas GN, Toulis KA, et al. Metabolically healthy obese and incident cardiovascular disease events among 3.5 million men and women. J Am Coll Cardiol 2017; 70: 1429-37.

23. Shubair MM, Kodis J, Mc Kelvie RS, Arthur HM, Sharma AM. Metabolic profile and exercise capacity out- 
comes: their relationship to overweight and obesity in a Canadian cardiac rehabilitation setting. J Cardiopulm Rehabil 2004; 24: 405-13.

24. Katzmarzyk PT, Church TS, Janssen I, Ross R, Blair SN. Metabolic syndrome, obesity, and mortality: impact of cardiorespiratory fitness. Diabetes Care 2005; 28: 391-7.

25. Warburton DE, Nicol CW, Bredin SS. Health benefits of physical activity: the evidence. CMAJ 2006; 174: 801-9.

26. Shaw LJ, Berman DS, Hendel RC, et al. Cardiovascular disease risk stratification with stress single-photon emission computed tomography technetium-99m tetrofosmin imaging in patients with the metabolic syndrome and diabetes mellitus. Am J Cardiol 2006; 97: 1538-44.

27. De Lorenzo A, Glerian L, Amaral AC, Reis TB, Lima RSL. "Metabolically healthy" obesity: prevalence, clinical features and association with myocardial ischaemia. Obes Res Clin Pract 2017; 11: 315-23.

28. Bader DS, Maguire TE, Spahn CM, O`Malley CJ, Balady GJ. Clinical profile and outcomes of obese patients in cardiac rehabilitation stratified according to National Heart, Lung, and Blood Institute criteria. J Cardiopulm Rehabil 2001; 21: 210-7.

29. Shubair MM, Kodis J, Mc Kelvie RS, Arthur HM, Sharma AM. Metabolic profile and exercise capacity outcomes: their relationship to overweight and obesity in a Canadian cardiac rehabilitation setting. J Cardiopulm Rehabil 2004; 24: 405-13.

30. Katzmarzyk PT, Church TS, Janssen I, Ross R, Blair SN. Metabolic syndrome, obesity, and mortality: impact of cardiorespiratory fitness. Diabetes Care 2005; 28: 391-7.

31. Bourque JM, Beller GA. Stress myocardial perfusion imaging for assessing prognosis: an update. JACC Cardiovasc Imaging 2011; 4: 1305-19.

32. Johnson NP, Wy E, Bonow RO, Holly TA. Relation of exercise capacity and body mass index to mortality in patients with intermediate to high risk of coronary artery disease. Am J Cardiol 2008; 102: 1028-33.

33. Doehner W, Clark A, Anker SD. The obesity paradox: weighing the benefit. Eur Heart J 2010; 31: 146-8.

34. Lavie CJ, Mc Auley PA, Church TS, Milani RV, Blair SN. Obesity and cardiovascular diseases: implications regarding fitness, fatness, and severity in the obesity paradox. J Am Coll Cardiol 2014; 63: 1345-54.

35. Emerging Risk Factors Collaboration. Wormser D, Kaptoge S, Di Angelantonio E, et al. Separate and combined associations of body-mass index and abdominal adiposity with cardiovascular disease: collaborative analysis of 58 prospective studies. Lancet 2011; 377: 1085-95.

36. Kramer CK, Zinman B, Retnakaran R. Are metabolically healthy overweight and obesity benign conditions? A systematic review and meta-analysis. Ann Intern Med 2013; 159: 758-69.

37. Bell JA, Kivimaki M, Hamer M. Metabolically healthy obesity and risk of incident type 2 diabetes: a metaanalysis of prospective cohort studies. Obes Rev 2014; 15: 504-15.

38. Appleton SL, Seaborn CJ, Visvanathan R, et al. Diabetes and cardiovascular disease outcomes in the metabolically healthy obese phenotype: a cohort study. Diabetes Care 2013; 36: 2388-94

39. Malnick SDH, Knobler $\mathrm{H}$. The medical complications of obesity. Q J Med 2006; 99: 565-79.

40. Wildman RP, Muntner P, Reynolds K, et al. The obese without cardiometabolic risk factor clustering and the normal weight with cardiometabolic risk factor clustering: prevalence and correlates of 2 phenotypes among the US population (NHANES 1999-2004). Arch Intern Med 2008; 168: 1617-24.

41. Sliwinska A, Kasinska MA, Drzewoski J. MicroRNAs and metabolic disorders - where are we heading? Arch Med Sci 2017; 13: 885-96. 